\title{
Permanence de l'exploitation agricole familiale, une approche gestionnaire
}

Mohamed Gafsi ${ }^{1}$

\section{Introduction}

La notion d'exploitation agricole familiale a marqué fortement l'agriculture française pendant ces soixante dernières années. Au niveau politique comme professionnel, elle a été le moteur de la modernisation de l'agriculture, comme en témoigne la place qui lui a été réservée dans les lois d’orientation agricole de 1960 et de 1962, ainsi que dans les diverses lois suivantes. Au niveau scientifique, elle n’a pas cessé de faire l'objet de débats et de controverses entre les chercheurs qui ont accompagné l'évolution du monde agricole depuis la Libération. On débat régulièrement de la conception de l'exploitation agricole et de la place qu'elle occupe dans le paysage professionnel agricole. Si l'on considère qu'elle a été le modèle d'organisation socioéconomique qui a accompagné la modernisation de l'agriculture, les évolutions importantes du contexte agricole de ces dernières décennies ont fait émerger de nouveau des débats sur la nature de l'exploitation agricole (Barthélemy, 1988 ; Muller et al., 1989) et sur la pertinence de la référence à la notion d'exploitation agricole familiale.

En effet, le contexte socioéconomique des exploitations agricoles a fortement évolué ces dernières décennies (Desriers, 2007 ; Ambiaud, 2011). Le nombre d'exploitations ne cesse de diminuer; de 2,3 millions d'exploitations en 1955 à environ 500 mille exploitations en 2010, selon les sources de la statistique agricole. Cette évolution s'est traduite globalement par les éléments structurants suivants : une forte concentration (augmentation de la taille des exploitations, augmentation du niveau de capitalisation), une spécialisation et intensification des systèmes de production, une importante augmentation des formes sociétaires et du travail salarié. Ces transformations sont dues au processus de modernisation de l'agriculture entrepris depuis l'après-guerre et qui a abouti à ce qui est appelé le modèle productiviste. Elles touchent à la fois les structures des exploitations agricoles et leurs caractéristiques

1. ENFA, UMR Dynamiques Rurales, 31326 Castanet-Tolosan, France,

mohamed.gafsi@educagri.fr

Ce chapitre de l'ouvrage L'agriculture en famille : travailler, réinventer, transmettre est publié en Open Access sous licence creative commons CC-BY-NC-ND permettant l'utilisation non commerciale, la distribution, la reproduction du texte, sur n'importe quel support, à condition de citer la source. 
familiales (Drouet et al., 2005 ; Cochet, 2008). Ainsi les contours traditionnels de l'exploitation agricole familiale éclatent et des auteurs proposent d'autres notions, comme celle de l'entreprise agricole (Barthélemy, 1988 et 1997) ou celle d'exploitation post-familiale (Rémy, 2013), pour qualifier les nouvelles structures de la production agricole. Dans ce contexte, on se pose la question de la pertinence de la référence à la notion d'exploitation agricole familiale pour saisir la réalité complexe des structures de production agricole.

Dans ce chapitre, nous considérons que, bien que fortement questionné par les transformations structurelles du contexte agricole, le modèle de l'exploitation familiale continue à être pertinent pour appréhender le fonctionnement des exploitations agricoles. La permanence de ce modèle est conditionnée par la capacité d'adaptation et d'évolution de l'exploitation agricole en tant que structure organisationnelle. Nous souhaitons apporter un éclairage à partir d'une approche gestionnaire qui fait référence à deux notions fondamentales (Hatchuel, 2000) : la complexité d'une organisation et la dialectique transformation - continuité de cette organisation. En utilisant une grille d'analyse mobilisant ces deux notions, nous revisitons l'évolution des exploitations agricoles au long des soixante dernières années pour montrer tout d'abord, les différentes figures de transformation de ces exploitations, en fonction des défis auxquels elles ont été soumises, tout en conservant leur identité d'exploitation familiale. Nous discutons ensuite la thèse de la permanence de l'exploitation familiale. Comment les sciences de gestion ont-elles conceptualisé l'objet de l'exploitation agricole familiale et comment peuvent-elles aider à comprendre ses transformations?

Dans un premier temps, nous présentons le cadre analytique en précisant, tout d'abord, la nature et les caractéristiques des organisations en général, en tant qu'objet des sciences de gestion, puis la conception de l'exploitation agricole en particulier. Nous abordons, par la suite, les premiers travaux des gestionnaires pour définir la nature et le fonctionnement de l'exploitation agricole, ainsi que les évolutions des cadres analytiques des chercheurs pour saisir et accompagner les différentes transformations de ces exploitations. Ces transformations sont en lien avec les principaux enjeux qu'ont connu les exploitations et que l'on peut résumer de manière globale aux trois suivants : la modernisation de l'agriculture survenue dans les trente glorieuses, la contestation du modèle productiviste des années 1980 et 1990, puis le nouveau contrat social pour une agriculture durable et multifonctionnelle des années 2000. Nous discutons, dans un troisième temps, la thèse de la permanence du modèle familial, ainsi que la pertinence de la réapparition de la référence au modèle de l'entreprise ces dernières années, eu égard des enjeux actuels de l'agriculture.

\section{L'exploitation agricole, une organisation complexe}

La littérature gestionnaire considère que l'organisation, au sens d'un collectif historiquement repérable (Sorge, 1997), constitue l'objet des sciences de gestion. Bien que l'histoire de la gestion ait été très liée à l'histoire des entreprises (Bouilloud et 
Lecuyer, 1994), ces dernières ne représentent que l'un des types d'organisations, à savoir l'organisation privée à but lucratif. Mais au-delà de la précision que l'organisation est l'objet générique des sciences de gestion, ce qui est important c'est comment ces derniers formalisent la nature et le fonctionnement de l'organisation. Hatchuel (2000) précise la nature de cette organisation : c'est une action collective artéfactuelle, une construction sociale et non un phénomène naturel ou une donnée anthropologique. L'auteur apporte deux fondements théoriques importants dans l'approche gestionnaire : (i) la nature complexe de l'organisation dans laquelle s'entremêlent plusieurs dimensions à la fois. "L'entreprise constitue un type d'action collective où les phénomènes économiques et sociaux s'emmêlent inévitablement " (p. 16). Par conséquent, les sciences de gestion composent avec cette complexité et elles sont donc de nature interdisciplinaire. (ii) En tant que construction sociale, l'entreprise jouit d'une liberté inédite : "il lui revient de définir ce qu'elle va faire et la manière dont cette définition sera conduite ". De ce fait, l'entreprise peut connaître des évolutions et des métamorphoses sans perdre son identité. Plus encore, selon cet auteur, « la révision de ses frontières (physiques, légales, humaines, commerciales, etc.) est une condition de son existence " (ibid).

Attardons-nous un peu sur ces deux éléments fondamentaux des sciences de gestion. Concernant le premier élément, en affirmant l'imbrication du social et de l'économique, l'auteur s'appuie sur l'une des conclusions mise en avant par le développement successif des théories des organisations (Rojot et Bergman, 1989 ; Plane, 2000). En effet le développement a mis en évidence le caractère multidimensionnel de l'entreprise et sa nature complexe. La seule référence à la conception positiviste et rationaliste, qui considère l'organisation comme une machine (Morgan, 1989) dont on peut organiser et déterminer le fonctionnement de manière scientifique est très réductrice. Ceci est important dans la mesure où toute modélisation de l'entreprise contient en elle-même des hypothèses sur son fonctionnement. Une approche réductrice aboutit à une image partielle de la réalité de cette entreprise. C'est ce qui a été reproché à l'approche classique des organisations représentée par les travaux de F.W. Taylor (1911) et de H. Fayol (1918).

Le deuxième élément important est relatif au processus d'adaptation et de métamorphose de l'entreprise, qui lui permet d'assurer sa pérennité : liberté et exigence à la fois de réviser ses frontières et son fonctionnement pour continuer à exister. La notion de pérennité peut avoir deux acceptions (Mignon, 2009) : premièrement, patrimoniale qui consiste à faire perdurer un patrimoine et à pérenniser le contrôle de l'entreprise par le même groupe de propriétaires (une famille dans le cas d'une exploitation agricole familiale) ; deuxièmement, organisationnelle qui revient à faire vivre un projet global, dans la durée, l'entreprise en tant qu'organisation. Patrimoniale ou organisationnelle, la pérennité de l'entreprise dépend de sa capacité à faire face, au cours de son histoire, à des bouleversements externes ou internes tout en préservant l'essentiel de son identité. Ainsi la dialectique entre changement et continuité constitue le cœur de la problématique de pérennité. Par ailleurs, plusieurs courants de sciences de gestion ont traité cette problématique, parmi lesquels on peut citer le courant de l'apprentissage et du changement organisationnel (Argyris et Schon, 2002). La référence à la notion de pérennité dans 
notre cas couvre les deux aspects : celui patrimonial qui renvoie au contrôle familial sur l'exploitation en tant qu'élément patrimonial, et celui organisationnel qui fait référence à la flexibilité de l'exploitation (Chia et Marchesnay, 2008).

Partant de cette grille d'analyse, l'approche gestionnaire considère que l'exploitation agricole est une organisation complexe qui comporte plusieurs dimensions fortement interconnectées. On peut citer les dimensions économique, patrimoniale, sociale, territoriale, etc. Le caractère familial, souvent souligné dans les travaux de sociologues (Barthez, 1982 ; Lamarche, 1991 ; Ait Abdelmalek, 2000), est l'un des éléments de cette complexité qui empreigne le fonctionnement de l'exploitation. Ce caractère peut être plus ou moins affirmé selon les types d'exploitations. Pour assurer son existence, l'exploitation agricole en tant qu'organisation est en perpétuel processus d'adaptation aux évolutions socioéconomiques. Elle prend donc différentes configurations dans le cadre de ces transformations. Nous tenterons d'analyser dans la partie suivante, le processus d'adaptation de l'exploitation agricole et ses différentes configurations.

\section{L'exploitation agricole familiale : naissance et évolution}

Avant d'être un objet scientifique, la notion d'exploitation agricole a été une construction sociale et politique. Son émergence date de la fin du XIX ${ }^{\mathrm{e}}$ siècle, notamment avec les enquêtes des statistiques agricoles (Laurent et Rémy, 2000). C'est dans les années 1960 qu'il y a eu réellement débat et formalisation de la notion d'exploitation agricole familiale, non sans controverse. Ce débat scientifique a été soutenu par le défi de la modernisation de l'agriculture et par la volonté politique de promouvoir le modèle de l'exploitation familiale.

Promue et instituée comme modèle d'organisation de la production agricole, l'exploitation agricole familiale va connaître des transformations importantes en lien avec des défis et des remises en question. De ce processus d'adaptation naissent différentes configurations d'exploitations familiales.

\section{Naissance dans la controverse : entreprise agricole ou exploitation familiale?}

Les premières formalisations gestionnaires de la notion d'exploitation agricole familiale en France ont été, dans un contexte de forte controverse entre deux visions opposées, celle de l'entreprise agricole issue du monde industriel et celle de l'exploitation familiale qui s'appuie sur les spécificités agricoles. Mais avant de développer ces approches, soulignons que les travaux des chercheurs étaient fortement en lien avec le grand mouvement politique et socioprofessionnel de modernisation de l'agriculture. 
En effet, la démarche volontariste de modernisation de l'agriculture, entreprise dans les années 1950 et 1960, a fortement bousculé le paysage agricole marqué par une agriculture traditionnelle. Elle s'est traduite par l'application de nouvelles méthodes de production basées sur les progrès techniques et la mécanisation, et par l'intégration des exploitations au marché. L'exploitation agricole familiale modernisée a succédé à une petite agriculture traditionnelle, peu insérée au marché, qualifiée de paysanne. Inlassablement promue dans le discours politique, économique et syndical, elle a été consacrée comme modèle canonique de l'organisation de la production agricole (Rémy, 2013). Les exploitants agricoles modernistes étaient à la tête de ce mouvement de transformation de l'agriculture. On le voit, la notion d'exploitation agricole familiale s'est imposée dans les faits comme moteur de la modernisation et par-là même comme objet des débats vifs entre les scientifiques. La première approche des gestionnaires ruraux (Chombart de Lauwe et al., 1963) se référait au modèle général de l'entreprise, issu du monde industriel, pour comprendre ce nouvel objet qui est l'exploitation agricole moderniste et proposer des méthodes scientifiques pour sa gestion.

\section{La nouvelle entreprise agricole}

Justement la figure de l'entreprise ${ }^{2}$ est employée dans les débats des années 1960, comme en témoignent les propositions des tenants de la "nouvelle entreprise agricole ". Celle-ci est définie comme " un ensemble d'ateliers de production placés sous l'autorité d'un chef » (Gervais et al. 1965, p. 115). Elle représente les exploitations agricoles modernes. Pour les promoteurs de la modernisation, la tentation est grande de voir l'agriculture suivre les mêmes principes et méthodes industriels. Ils prônent alors « l'industrialisation de l'agriculture " qui consiste, selon eux, en " une approche scientifique de l'agriculture " (Chombart de Lauwe, 1969).

Partant de cette approche, Chombart de Lauwe et al. (1963) proposent un modèle de gestion de l'exploitation agricole reposant sur une approche normative, inspiré des travaux développés aux États-Unis au début du XX $X^{\mathrm{e}}$ siècle (Laurent et al., 2003). L'exploitation agricole est considérée comme une entreprise au même titre que les autres, qui combine des facteurs de production en vue de réaliser un profit, et à laquelle il faut appliquer les mêmes méthodes d'organisation scientifique du travail. Les auteurs proposent un ensemble d'outils et de méthodes permettant de déterminer le système de production optimal, à travers la définition des « combinaisons rentables ", et une conduite rationnelle de l'exploitation agricole. Cette approche a eu un succès énorme, dans les années 1960, dans les cercles de l'enseignement et du développement agricoles. Elle s'est confortée par la suite avec le développement de la comptabilité dans les exploitations agricoles et la création des CER (centres d'économie rurale) (Marshall, 1984). Elle a aussi marqué les travaux scientifiques de l'école de gestion de Grignon (Cordonnier et al., 1970 ; Carles, 1990) et les discours de certains professionnels agricoles, notamment des conseillers de gestion.

2. Figure se référant à une approche mécaniste de l'entreprise dont le fonctionnement pouvait être déterminé de manière rationnelle et scientifique. 
Mais cette première approche a généré des critiques en lien avec la conception industrielle de l'exploitation agricole, en tant qu'objet de gestion. En effet, les transformations produites par la modernisation agricole et la référence au modèle rationaliste de l'entreprise ont suscité la crainte de voir installer une agriculture d'entreprise, basée sur le pouvoir du capital et mue par le seul profit. Gervais et al. (1965) montrent que dans ce contexte, on s'oriente inéluctablement vers une " agriculture sans paysans " qui ferait des agriculteurs, les salariés d'un chef d'entreprise détenteur de capitaux. Une seconde critique radicale vient des tenants de la spécificité de l'agriculture qui justifient les nombreuses et fortes interventions publiques en agriculture (Petit, 2006). En lien avec ces spécificités, Petit (1975) soulignait la nécessité de prendre en compte le caractère familial des exploitations agricoles pour comprendre l'évolution de l'agriculture en France.

\section{Le système exploitation-famille}

La seconde approche des gestionnaires, développée par des chercheurs dijonnais s'inscrit pleinement dans la spécificité de l'agriculture (Petit, 1975 ; Brossier et al., 1997). Elle s'oppose à l'approche développée par Chombart de Lauwe et son équipe, à la fois au niveau de la conception de l'exploitation et des méthodes de sa gestion.

L'exploitation agricole ne peut être assimilée à une entreprise capitaliste que l'on peut gérer indépendamment des considérations familiales. Le lien avec la famille est fondamental pour comprendre la logique de fonctionnement de l'exploitation agricole. Cette conception de l'exploitation s'appuie sur les travaux de Tchayanov (1925), qui met en avant l'importance du caractère familial, et des sociologues sur les relations entre famille et exploitation (Barthez, 1982). Elle a été formalisée par la suite par le concept de "système exploitation-famille " en adoptant une approche systémique de l'exploitation agricole (Osty, 1978). L'exploitation agricole n'est pas une simple juxtaposition d'ateliers spécialisés de production, mais un système complexe et un projet finalisé de la famille (Brossier et al., 1997). Cette conception systémique considère l'exploitation dans sa globalité. Elle a été développée aussi sous le nom de "l'approche globale de l'exploitation agricole " (Bonneviale et al., 1989) qui a fortement marqué l'enseignement agricole et en partie le monde du développement depuis le début des années 1980.

En ce qui concerne les méthodes de gestion, les gestionnaires de l'école de Dijon se sont appuyés sur les travaux du courant behavioriste (March et Simon, 1964 ; Cyert et March, 1970) et le concept de la rationalité limitée. Ils ont développé une approche compréhensive de gestion de l'exploitation agricole ; une approche « centrée sur l'acteur » et la prise de décision (Petit, 1975 ; Brossier et al., 1997). Les agriculteurs " ont de bonnes raisons de faire ce qu'ils font"; ils ne suivent pas forcément et uniquement un objectif de profit ; ils n'ont pas un comportement d'optimisation mais ils cherchent un compromis entre plusieurs objectifs. Ces objectifs sont étroitement liés aux finalités de la famille. Par conséquent, la famille qui détient les capitaux d'exploitation et qui fournit le travail, est fortement impliquée dans la gestion et le fonctionnement de l'exploitation, d'où les relations d'interdépendance entre les deux entités et le recours à la notion de système exploitation-famille pour saisir ces relations. 
Les travaux des gestionnaires de l'école de Dijon ont élaboré un cadre d'analyse permettant de saisir la complexité et les spécificités de l'exploitation agricole familiale. La notion d'exploitation agricole familiale est depuis largement admise et reconnue. Précisons tout de même que le triomphe de cette notion ne revient pas uniquement aux apports de l'approche des gestionnaires. Les travaux des économistes agricoles (Petit, 1975 ; Boussard, 1987) et des sociologues ruraux ont contribué à asseoir la notoriété de cette notion. C'est probablement à cause de la diversité de filiations de cette notion qu'il manque, malgré ce succès sans partage, une définition scientifique précise de ce qui est une exploitation agricole familiale. Certes le caractère familial de l'exploitation est bien mis en avant, mais on ne se tient presque qu'à cela. Les définitions avancées font généralement référence à l'économiste russe Tchayanov (1925). Or les travaux de celui-ci définissent l'exploitation paysanne familiale et non pas l'exploitation agricole familiale, dont l'intégration au marché n'est plus en question. Lamarche (1991) mentionne cette distinction et propose une définition de l'exploitation familiale : "unité de production agricole où propriété et travail sont intimement liés à la famille» (p. 10). Les travaux des gestionnaires (Brossier et al., 1997) rajoutent aux éléments évoqués par Lamarche - propriété et travail - la dimension décisionnelle : les stratégies et le pilotage de l'exploitation sont guidés par les finalités familiales. Avec ces trois caractéristiques, on a un cadre général de ce qui est une exploitation agricole familiale. Mais les débats sur une définition précise restent ouverts. À titre d'exemple, il y a une idée implicite, peu formalisée, qui considère que le travail sur l'exploitation doit être exclusivement familial. Dès qu'il y a un travail salarié permanent, le caractère familial est remis en cause ; on passerait alors à une " exploitation patronale" (Bélières et al., 2013).

Comme on vient de le voir, l'exploitation agricole familiale, en tant que notion scientifique, est née dans un contexte de controverse par opposition à la notion d'entreprise agricole défendue par les tenants de la thèse de l'industrialisation de l'agriculture. Cette notion dont les contours restent peu précis, fera l'objet de nouveaux questionnements à l'aune des évolutions des exploitations agricoles ces dernières décennies.

\section{Adaptations et transformations pour faire face aux défis}

Figure emblématique de la modernisation de l'agriculture, l'exploitation agricole familiale affrontera à partir de la fin des années 1970, deux principaux défis : l'un structurel en lien avec les évolutions internes dans les exploitations et dans les familles, l'autre est conjoncturel en lien avec les évolutions de l'environnement socio-économique. L'approche gestionnaire apporte des outils pour comprendre les adaptations et les transformations de l'exploitation agricole face à ces défis.

\section{Face au défi structurel, pluralité des formes d'exploitations familiales}

Le défi structurel est double; i a touchéà la fois l'exploitation et la famille. Concernant les exploitations, la modernisation a débouché sur le modèle productiviste caractérisé 
par la spécialisation, la capitalisation et la concentration des exploitations. En même temps, les évolutions structurelles touchent aussi l'organisation de la famille. On peut citer le développement des activités extérieures à l'exploitation pour les chefs d'exploitation comme pour les conjoints, les installations hors cadre familial, les transformations des rapports qu'entretiennent les familles au foncier, etc.

La modernisation et ses conséquences en termes d'évolutions structurelles ont donné lieu à une différentiation socioprofessionnelle entre différentes formes d'agricultures (Pernet, 1990) : allant d'une agriculture fortement modernisée à une agriculture traditionnelle réfractaire au progrès technique et à l'intégration au marché, en passant par des formes intermédiaires, qui ne peut pas ou ne veut pas adopter le modèle de l'agriculture modernisée. On compte ainsi parmi ces formes intermédiaires la figure qualifiée "d'agriculture différente " (Muller et al., 1984). Elle regroupe les différentes initiatives et formes de diversification agricole permettant de dégager un revenu suffisant et de se reproduire sans emprunter la voie du modèle productiviste. Pour saisir ces initiatives, les chercheurs proposent la notion « d'exploitation rurale " (Muller et al., 1989 ; Pernet, 1990) et en donnent les principaux traits : (i) mise en oeuvre de stratégies entrepreneuriales complexes, (ii) importance de la fonction commerciale et (iii) la compétence de l'exploitant rural est une compétence managériale.

L'exploitation agricole familiale couvre-t-elle l'ensemble de ces formes ? L'approche gestionnaire répond par l'affirmative et propose des outils pour comprendre la diversité des formes d'exploitations agricoles familiales. Déjà en 1975, Petit soulignait les transformations des exploitations agricoles et la capacité de celles-ci à absorber ces transformations. Il écrivait « le maintien des exploitations familiales ne signifie pas que celles-ci ne se soient pas profondément transformées... Ce qui est le plus remarquable ce n'est peut-être pas le maintien des exploitations familiales mais la plasticité de cette forme d'unité de production » (p. 46). L'auteur donne les principales caractéristiques de l'exploitation agricole familiale : plasticité, adaptation en permanence, diversité de logiques de fonctionnement interne. Les travaux des chercheurs de Dijon, conduits par la suite, ont élaboré des outils analytiques permettant de comprendre les dynamiques et les formes d'exploitations familiales. On peut citer la théorie du comportement adaptatif (Petit, 1981 ; Brossier et al., 1991) qui vise à comprendre le fonctionnement des exploitations agricoles dans une approche dynamique (voir chapitre de Chia et al. dans cet ouvrage). Elle repose sur le postulat de cohérence du comportement des agriculteurs et le mouvement de la double adaptation : changement de la situation de l'exploitation et modification des finalités de l'agriculteur et sa famille. Le deuxième outil analytique est celui de typologie de fonctionnement (Brossier et Petit, 1977 ; Brossier et al., 1997) qui met l'accent sur la diversité des logiques d'action des agriculteurs selon leurs projets et situations. Il permet d'expliquer la diversité des formes d'agricultures qui sont certes en lien avec les caractéristiques structurelles des exploitations (dimension, orientation, capitalisation, etc.) mais aussi en fonction des projets et des finalités des agriculteurs. 


\section{Face à l'incertitude de l'environnement, la gestion stratégique de l'exploitation}

Les processus d'adaptation des exploitations agricoles familiales sont aussi en lien avec les évolutions de l'environnement. Longtemps protégée par les politiques publiques, l'exploitation agricole affronte, depuis la fin des années 1980, des évolutions de l'environnement socio-économique de plus en plus complexes : crise de surproduction, réformes successives de la PAC, ouverture de l'agriculture aux négociations de l'OMC (Organisation mondiale du commerce), etc. Les agriculteurs expriment des inquiétudes concernant la viabilité économique de leurs exploitations et la reproductibilité de leurs outils de travail. Dans ce contexte, les agriculteurs sont aussi très liés aux structures économiques de l'aval, d'où la nécessité d'un pilotage de l'exploitation par l'aval (Sebillotte, 1996). Les crises sanitaires à répétition des années 1990, ainsi que les problèmes écologiques, n’ont fait qu'augmenter le caractère incertain de l'environnement socio-économique des exploitations.

L'incertitude qui caractérise l'environnement des exploitants à la suite des différentes crises (économique, écologique, sanitaire, sociale) appelle à la gestion stratégique et aux capacités d'anticipation et d'adaptation. La question du raisonnement stratégique et de la compétence managériale ne concerne pas uniquement les agriculteurs innovateurs de "l'agriculture différente ". Elle s'est posée aussi pour les exploitants agricoles de l'agriculture moderne. Dans l'environnement stable des trente glorieuses, il était normal de ne se préoccuper que de la fonction de production, les prix et les débouchés étant garantis. Les recherches en gestion de l'exploitation ont peu abordé les dynamiques d'interaction entre l'exploitation et son environnement. Elles se sont plutôt centrées sur l'organisation et le fonctionnement interne de l'exploitation. Dans le nouveau contexte d'incertitude, nous avons proposé d'élargir le champ traditionnel, qui est l'exploitation-système (interactions exploitation-famille), pour prendre les relations avec l'environnement comme objet de recherche. Cela se traduit par « la vision de l'exploitation/ organisation active (relations)... qui dispose d'une autonomie d'action et valorise ses rapports avec l'environnement " (Gafsi, 1998, p. 255). D'autres travaux, conduits au début des années 1990, ont abordé aussi le questionnement de la gestion stratégique des exploitations agricoles (Attonaty et Soler, 1992). Hémidy et al. (1993) ont proposé des voies d'instrumentation et de pilotage stratégique dans l'exploitation agricole, dans une perspective de conseil de gestion. Par ailleurs, les problèmes de gestion stratégique n'étaient pas uniquement des questions de chercheurs; des acteurs professionnels se sont aussi interrogés sur ce domaine de gestion (Guichard et Michaud, 1994).

Globalement, l'exploitation agricole familiale partie intégrante du modèle socioprofessionnel issu de la modernisation a affronté plusieurs défis et évolutions. La notion même d'exploitation agricole familiale a été requestionnée par des chercheurs à la fin des années 1980. Blanc et al. (1990) s'interrogent "l'agriculture française est-elle encore familiale ? ». Barthélemy (1988) publiait " la naissance de l'entreprise agricole». Mais du point de vue des gestionnaires, l'exploitation agricole dispose d'une capacité d'adaptation qui lui a permis de composer avec les défis 
structurels et conjoncturels. Avec cette capacité d'adaptation, l'exploitation agricole familiale est-elle en mesure de relever le défi de la durabilité ?

\section{L'exploitation agricole à l'épreuve de la durabilité}

La question de la nature de l'exploitation agricole et la pertinence de la référence au modèle familial est à nouveau posée dans les années 2000. Les exploitations agricoles font face à nouveau à des incertitudes internes et externes dans un contexte global de questionnement et de débat sur un nouveau contrat social de l'agriculture (Landais, 1998). Ce nouveau contrat invite à une inscription territoriale de l'exploitation agricole et au développement d'une agriculture durable.

\section{L'exploitation agricole territoriale}

Malgré les différentes mesures, notamment les réformes successives de la PAC, les évolutions structurelles pointées précédemment ont continué mais avec un rythme ralenti par rapport aux décennies précédentes (Desriers, 2011). Le nombre d'exploitations ne cesse de diminuer ; la capitalisation et la spécialisation ont continué à progresser. L'agriculture s'est fortement inscrite dans une approche verticale de filières au détriment de son inscription territoriale et locale. La loi d'orientation agricole de 1999 s'est donné l'ambition de réorienter l'agriculture dans une perspective de durabilité et de la « re-territorialiser » (Gafsi, 2003). Elle a mis en place pour cet effet un outil contractuel, le CTE (contrat territorial d'exploitation). Comment saisir à partir d'une approche gestionnaire cette dimension territoriale du fonctionnement de l'exploitation agricole?

Nous avons proposé avec des collègues la notion d'ancrage territorial, employée pour traduire cette dimension (Gafsi et al., 2002 ; Nguyen et al., 2004). L'ancrage territorial d'une exploitation agricole est défini comme un "processus de valorisation, de préservation et de production de ressources spécifiques au territoire " (Gafsi, 2006, p. 494). Ces ressources territoriales couvrent les dimensions naturelle, relationnelle, organisationnelle, symbolique et économique. Il est important de souligner que cette définition de l'ancrage dépasse la vision classique de l'exploitation agricole, simple unité de production utilisatrice de facteurs de production génériques dont est doté l'espace où elle se trouve, pour s'interroger sur sa contribution à la production et à la préservation des ressources spécifiques à son territoire. Cette relation réciproque donne lieu, dans une perspective d'ancrage territorial, à des interdépendances multiples entre l'exploitation en tant qu'organisation et son environnement local (naturel, social et économique). Ce processus contribue à sceller les devenirs de l'exploitation et du territoire dans lequel elle s'inscrit. Partant de l'hypothèse que l'avenir des exploitations agricoles se joue de plus en plus dans des territoires construits à l'échelle locale, Vandenbroucke (2013) propose la notion « d'exploitation agricole territoriale » pour saisir les liens d'interdépendance exploitation agricole/territoire (voir le chapitre à ce sujet dans cet ouvrage). L'auteur la définit " comme une exploitation dont la viabilité repose sur son insertion dans des systèmes de coordination horizontaux, en relation avec des acteurs agricoles et non 
agricoles"(p. 99). Le développement récent de la commercialisation en circuits courts de proximité conforte l'ancrage territorial des exploitations agricoles (Mondy et al., 2012).

\section{L'exploitation agricole durable : approche par les ressources et flexibilité}

La volonté de reterritorialisation de l'agriculture s'inscrit dans l'orientation globale de développement d'une agriculture durable et multifonctionnelle. Suite à la loi d'orientation agricole de 1999, beaucoup d'exploitations ont saisi l'opportunité et se sont engagées dans des CTE, certaines pour réaliser la reconversion à l'agriculture biologique, laquelle a connu un important développement. Mais cette dynamique a été stoppée avec l'alternance politique en 2002. On observe, en revanche, le retour de l'esprit de modernisation dans les lois agricoles de 2006 et 2010, sous l'angle du soutien de la compétitivité des exploitations agricoles. Ainsi, la loi d'orientation de 2006 a créé le fonds agricole et mentionne dans son premier chapitre l'objectif de "faire évoluer l'exploitation agricole vers l'entreprise agricole ". Mais malgré ce renoncement, la problématique de la durabilité de l'agriculture est restée toujours bien présente. Comment les sciences de gestion ont-elles abordé l'adaptation des exploitations agricoles aux injonctions de développement de systèmes durables?

Les travaux développés ont approfondi les précédentes orientations de gestion stratégique de l'exploitation agricole. En effet, l'orientation vers une agriculture durable induit pour les exploitations agricoles des changements d'orientations stratégiques. C'est surtout le cas pour la majorité des exploitations spécialisées qui s'inscrivent encore dans le modèle productiviste. Ces changements se traduisent par de nouvelles rationalisations quant au choix des ressources stratégiques pour l'activité de ces exploitations et aux combinaisons rentables des ressources. En s'appuyant sur la théorie des ressources (Barney, 1991 ; Wernerfelt, 1995) nous avons proposé de revisiter l'approche classique de l'exploitation agricole pour proposer un cadre analytique permettant d'intégrer les enjeux de la durabilité. Le modèle proposé comprend cinq types de capitaux : en ajoutant aux trois capitaux communément pris en compte (physique, financier et humain), le capital naturel et le capital social (Gafsi, 2006). Dans une perspective de durabilité, l'exploitation utilise non seulement les ressources relevant des cinq capitaux, mais elle contribue aussi à la préservation et/ ou à la création de ces ressources. Il s'agit là d'un changement important dans la logique d'action des agriculteurs : d'une logique centrée uniquement sur l'utilisation des ressources selon le schéma simple de maximisation à court terme de la fonction de production, à une logique fondée sur la dynamique récursive de valorisation, de préservation et de création des ressources. Cette logique rejoint la notion de «performance globale " proposée par Reynaud (2006). Le modèle d'exploitation agricole durable proposé est mobilisé dans les travaux récents sur la durabilité des exploitations de l'agriculture biologique, ainsi que dans l'élaboration de méthode d'évaluation de la durabilité de l'exploitation agricole (la méthode IDEA ${ }^{3}$ par

3. Indicateurs de durabilité des exploitations agricoles; voir www.idea.portea.fr 
exemple). D'autres travaux récents ont mis l'accent sur les capacités d'adaptation et la flexibilité des exploitations agricoles pour améliorer leur durabilité (Dedieu et al., 2008 ; Darnhoefer et al., 2010).

Face au défi de la reterritorialisation et de la durabilité, les travaux des gestionnaires ont souligné les capacités d'adaptation de l'exploitation agricole familiale moyennant la révision à la fois de son fonctionnement et de ses frontières. La prise en compte de l'inscription territoriale dans son fonctionnement aboutirait à une autre forme d'exploitation agricole qualifiée de " territoriale ». L'approche stratégique par les ressources permet de renouveler le cadre d'analyse de l'exploitation agricole pour saisir les enjeux de la durabilité.

\section{Les limites de la permanence}

L'exploitation agricole familiale a subi des évolutions importantes. Mais finalement, force est de constater aujourd'hui la permanence des exploitations familiales. Les sciences de gestion expliquent cette permanence par la capacité d'adaptation de l'exploitation en tant qu'organisation, et sa métamorphose. Mais jusqu'où peut aller cette capacité d'adaptation ? La permanence de l'exploitation agricole familiale a-telle des limites?

\section{Pérennité de l'exploitation agricole : métamorphoses et continuité}

Nous avons souligné plus haut que l'approche gestionnaire considère que l'exploitation agricole comme une organisation complexe, devrait assurer sa pérennité par ses processus d'adaptation. La pérennité d'une organisation signifie la conciliation de deux exigences contradictoires : changement et continuité. L'analyse globale de l'évolution de l'exploitation agricole montre que celle-ci a pu concilier les deux exigences.

Le changement, on l'a vu, se situe à la fois au niveau de l'exploitation et de la famille. Le portrait des exploitations familiales d'aujourd'hui est largement différent de celui des années 1960. L'exploitation agricole s'est métamorphosée à maintes reprises pour s'adapter aux évolutions structurelles et conjoncturelles. La révision successive de ses frontières a donné lieu à plusieurs formes d'exploitations. Ainsi, nous avons assisté à l'émergence, au fil du temps, de différentes dénominations qui qualifient ces formes (Fig. 1) : système exploitation-famille, exploitation rurale, exploitation territoriale, exploitation durable. Cette diversité de formes reflète le potentiel intégrateur du modèle de l'exploitation agricole familiale, qui permet de rendre compte de la pluralité des agricultures. 


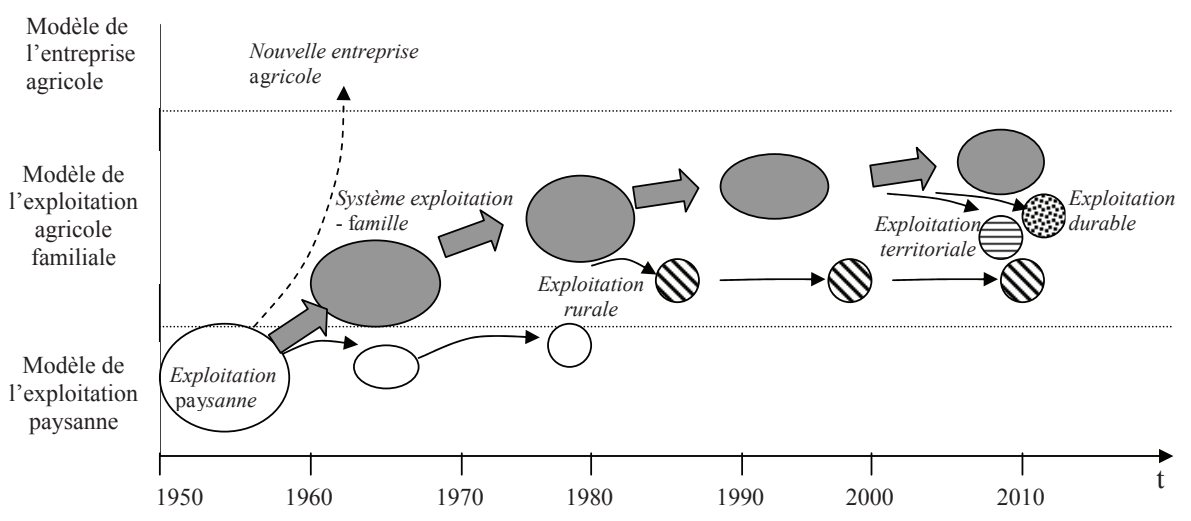

Fig. 1. Trajectoires d'évolution des exploitations agricoles.

Les transformations subies ont sans doute modifié le fonctionnement des exploitations, l'organisation du travail, la conception du métier, etc. Mais ces exploitations ont-elles perdu leur identité familiale ? Nous avons évoqué plus haut les trois éléments qui définissent le caractère familial de l'exploitation : propriété du capital, travail et pouvoir décisionnel. L'analyse du portrait des exploitations aujourd'hui, dans leur diversité, montre que ces traits fondamentaux ont été préservés. Le capital d'exploitation reste la propriété de la famille. En 2010, 69 \% des exploitations agricoles ont un statut juridique d'exploitation individuelle. Le statut de sociétés commerciales et coopératives ne représente que $1 \%$ (Agreste, 2014). Même si le statut des EARL (exploitation agricole à responsabilité limitée) permet de séparer le patrimoine professionnel du patrimoine personnel, dans les faits, le développement de cette forme sociétaire est davantage motivé par l'optimisation fiscale et les avantages sociaux et reste sans incidence sur la gestion patrimoniale. Bessière et al. (2011) ont mis en évidence l'importance des mécanismes de transmission intergénérationnelle du patrimoine productif agricole, en particulier le foncier. Concernant le travail, même s'il y a progression du travail salarié, le travail familial reste un élément important dans l'exploitation. En 2010, seuls $14 \%$ des exploitations en France recourent à la main-d'œuvre permanente non familiale. Dans les moyennes et grandes exploitations, qui recourent le plus au travail salarié (permanent et temporaire), la part de celui-ci représente $30 \%$ du volume total (Agreste, 2013). La famille, à travers au moins le chef d'exploitation quand il y a recours au salariat, est toujours impliquée dans la réalisation du travail et la conduite effective de l'exploitation. Notons que le travail réalisé par les ETA (entreprise de travaux agricoles) et les CUMA (Coopérative d'utilisation de matériel agricole) ne représente que 1 à $3 \%$ $\mathrm{du}$ volume total, selon la forme juridique de l'exploitation (Agreste, 2014). Avec les évolutions sociales, il est maintenant rare d'avoir l'implication de tous les membres de la famille dans le travail. En revanche, plus les membres de la famille sont impliqués dans le travail, plus le caractère familial de l'exploitation est renforcé. Enfin, en lien avec la propriété du patrimoine productif et le travail effectif, la dimension familiale marque fortement les grandes décisions de l'exploitation (Brossier et al., 
1997). Les orientations de l'exploitation continuent à être en lien direct avec les projets familiaux (besoins familiaux, transmission de l'exploitation, gestion de patrimoine). Après avoir posé la question « l'agriculture française est-elle encore familiale ? ", Blanc et al. (1990) concluaient "si les familles vivant sur les exploitations sont de moins en moins agricoles, on n'en conclura cependant pas que l'agriculture, elle, devient de moins en moins familiale» (p. 323).

$\mathrm{Au}$ vu de ces transformations et continuités, on peut conclure à la persistance de l'exploitation agricole familiale. Toutefois, la continuation du modèle de l'exploitation familiale sera-t-elle indéfinie ? Jusqu'où ce modèle pourrait-il repousser ses frontières sans perdre son identité ? La réponse n'est pas aisée tant la capacité de ce modèle à apporter des réponses inédites est grande. Mais on peut supposer que l'accumulation des transformations modernisatrices qui font perdre progressivement à la famille le contrôle du capital de l'exploitation conduirait à la fin de ce modèle. La question est donc à nouveau posée : quel est le devenir de l'exploitation agricole?

\section{L'exploitation agricole et le modèle d'entreprise}

Si les tendances agricoles actuelles continuent, on ne compterait en 2025 qu'environ 200000 exploitations spécialisées et intensives, largement intégrées dans un environnement concurrentiel, avec des conséquences environnementales et sociales catastrophiques (Gambino et Vert, 2012). Ces exploitations garderaient-elles un caractère familial ? S'oriente-t-on vers « l'exploitation post-familiale » (Rémy, 2013) ou rentre-t-on, cette fois-ci, dans le modèle de l'entreprise ? Si c'est le cas, quelle est la pertinence de ce modèle au regard des enjeux sociétaux actuels ?

Nous l'avons vu, dans une perspective gestionnaire, la référence au modèle de l'entreprise dans les années 1950 a suscité beaucoup de critiques. Et on le sait maintenant, cette conception rationaliste et déterministe de l'entreprise a été contestée et remise en cause à la fois pour les entreprises en général, avec les développements des théories des organisations et pour le secteur agricole en particulier. Les chercheurs en gestion de l'école de Grignon et les CER qui continuent à utiliser le qualificatif d'entreprise adhèrent-ils à la même conception de l'entreprise que les pionniers ? Probablement pas. Certes, ces scientifiques et professionnels s'inscrivent dans l'héritage de Chombart de Lauwe, mais les évolutions des travaux depuis la fin des années 1980 montrent une certaine rupture avec l'approche normative et rationnelle de l'entreprise. Le CER France propose une approche de «l'exploitation agricole flexible " basée sur un triptyque : projet patrimonial, projet technique et projet entrepreneurial (Séronie, 2007).

Dans les faits, depuis les années 1960, le modèle de l'exploitation familiale a triomphé en France. En revanche, dans les pays socialistes, la liquidation des structures familiales de production dans le cadre de la collectivisation générale " était une erreur stratégique majeure, ayant largement contribué plusieurs décennies plus tard à l'écroulement de ces régimes "(Petit, 2006, p. 487). Plus récemment, l'expérience du développement d'une agriculture de firme en Amérique latine (Guibert 
et al. 2011) a donné lieu à des conséquences désastreuses en termes d'exclusion sociale et de dégâts environnementaux (Tonneau et al., 2005). Or justement, c'est à cause de ce type de conséquences que les politiques publiques, en France, tentent depuis une quinzaine d'années de promouvoir une agriculture durable. Le devenir des exploitations agricoles est un enjeu important pour ces politiques publiques. N'oublions pas que le maintien des exploitations agricoles familiales dans les pays développés est dû, en partie, au soutien des pouvoirs publics qui sont depuis longtemps sous la tension entre l'impératif de soumettre l'agriculture aux mécanismes de la régulation marchande et le souci de protéger le revenu des agriculteurs (Petit, 2006).

Les tendances agricoles de fond et les évolutions prospectives mettent le modèle de l'exploitation familiale à rudes épreuves. De nouvelles configurations émergeront sans doute de ses dynamiques d'adaptation. L'ampleur des transformations fera sortir probablement les exploitations du modèle d'exploitation familiale. Mais cette évolution ne sera pas sans lien avec les politiques publiques et la place de l'agriculture dans la société de demain.

\section{Conclusion}

L’exploitation agricole familiale a été institutionnalisée depuis le milieu du XXe siècle. Bénéficiant de l’appui des pouvoirs publics, elle a été le moteur de la modernisation et du développement de l'agriculture. Les grandes transformations de l'agriculture et de la structure de la famille ont amené à poser la question de la pertinence de la notion de l'exploitation familiale aujourd'hui.

Nous avons montré dans ce chapitre comment les sciences de gestion ont appréhendé la naissance de la notion d'exploitation agricole familiale dans un contexte de controverse sur la nature de l'organisation de la production agricole. Considérant l'exploitation agricole comme une organisation complexe, les travaux des gestionnaires ont proposé des cadres analytiques pour comprendre les différentes évolutions des exploitations agricoles. On peut citer la théorie du comportement adaptatif, les typologies de fonctionnement, les méthodes de gestion stratégique, les approches d'analyse de l'ancrage territorial et de la durabilité des exploitations, etc. Selon l'approche gestionnaire, la thèse de la permanence de l'exploitation agricole familiale est au prix des transformations et des révisions de ses frontières, permettant d'intégrer différentes formes d'exploitations agricoles. Mais jusqu'à quelle limite l'exploitation agricole familiale peut-elle composer avec les transformations tout en préservant son identité ? Le devenir de l'exploitation agricole est une question importante des politiques publiques et des orientations que l'on souhaite donner à l'agriculture.

L'importance d'une approche gestionnaire vient de sa capacité heuristique à saisir la complexité de l'exploitation, en tant qu'organisation économique et patrimoine identitaire familial. Elle invite à une prise de recul et un élargissement de l'approche d'analyse des comportements des agriculteurs, qui ne sont pas exclusivement des 
actes économiques. La rationalité économique et cartésienne reste impuissante pour comprendre ces comportements. Il faut sans doute envisager une autre forme de rationalité (" humaine» ?) pour rendre intelligible les encastrements mutuels de l'économique, du social et de l'écologique. Dans ce cas, la voie vers la durabilité, on l'espère, sera plus aisée.

\section{Bibliographie}

Agreste, 2013, L'agriculture, la forêt et les industries agroalimentaires, GraphAgri 2013.

Agreste, 2014, Le statut juridique des exploitations agricoles : évolutions 1970-2010, Agreste Les Dossiers, 20.

Ait Abdelmalek, A., 2000, L'exploitation familiale agricole : entre permanence et évolution, Économie Rurale, 255-256, 40-52.

Ambiaud, E., 2011. Diversité du monde agricole, Analyse, 32, Publication du Centre d'études et de prospective, ministère de l'Agriculture.

Argyris, C., Schon, D., 2002. Apprentissage organisationnel : théorie, méthode, pratique, Bruxelles, DeBoeck Université.

Attonaty, J.-M., Soler, L.-G., 1992. Aide à la décision et gestion stratégique : un modèle pour l'entreprise agricole, Revue Française de Gestion, 89, 45-54.

Barney J., 1991. Firm resources and sustained competitive advantage, Journal of Management, 17, 99-120.

Barthélemy, D., 1988. La naissance de l'entreprise agricole, Paris, Economica.

Barthélemy, D., 1997. Evvaluer l'entreprise agricole, Paris, Presses Universitaires de France.

Barthez, A., 1982, Famille, travail et agriculture, Paris, Economica.

Bélières, J.-J., Bonnal, P., Bosc, P.-M., Losch, B., Marzin, J., Sourisseau, J.-M., 2013. Les agricultures familiales du monde : définitions, contributions et politiques publiques, Rapport Cirad, $1^{\text {re }}$ partie.

Bessière, C., De Paoli, C., Gouraud, B., Roger, M., 2011. Les agriculteurs et leur patrimoine : des indépendants comme les autres ? Économie et Statistique, INSEE, 444-445, 55-74.

Blanc, M., Brun, A., Delord, B., Lacombe, P., 1990. L'agriculture française est-elle encore familiale ?, in Coulomb, P., Delorme, H., Hervieu, B., Jollivet, M., Lacombe, P. (dir), Les agriculteurs et la politique, Paris, Presse de la Fondation nationale des sciences politiques, 310-327.

Bonneviale, J.-R., Jussiau, R., Marshal, E., 1989. Approche globale de l'exploitation agricole. Dijon, Édition INRAP.

Bouilloud, J.-P., Lecuyer, B.-P., 1994. Linvention de la gestion : Histoire et pratiques, Paris, L'Harmattan.

Boussard , J.-M., 1987. L'économie de l'agriculture, Paris, Economica. 
Brossier, J., Chia, E., Marshall, E., Petit, M., 1991, Gestion de l'exploitation agricole familiale et pratiques des agriculteurs. Vers une nouvelle théorie de la gestion, Canadian Journal of Agricultural Economics, 39, 2, 119-135.

Brossier, J., Chia, E., Marshall, E., Petit, M., 1997. Gestion de l'exploitation agricole familiale. Eléments théoriques et méthodologiques, Dijon, Cnerta.

Brossier, J., Petit, M., 1977. Pour une typologie des exploitations agricoles fondée sur les projets et situations des agriculteurs, Économie Rurale, 122, 34-43.

Carles, R., 1990. Le diagnostic financier de l'entreprise agricole, Paris, INRA Éditions.

Chia, E., Marchesnay, M., 2008. Un regard des sciences de gestion sur la flexibilité : enjeux et perspectives, in Dedieu, B., Chia, E., Leclerc, B., Moulin, C.-H., Tichit, M., L'élevage en mouvement : flexibilité et adaptation des exploitations d'herbivores, Paris, Quae, 23-36.

Chombart de Lauwe, J., 1969. Avons-nous une politique agricole aujourd'hui ? Économie Rurale, 79-80, 59-79.

Chombart de Lauwe, J., Poitevin, J., Tirel, J.-C., 1963. Nouvelles gestion des exploitations agricoles, Paris, Dunod.

Cochet, H., 2008. Vers une nouvelle relation entre la terre, le capital et le travail, Études foncières, 134, 24-29.

Cordonnier, P., Carles, R., Marsal, P., 1970. Économie de l'entreprise agricole, Paris, Cujas.

Cyert, R.-M., March, J.-G., 1970. Processus de la décision dans l'entreprise, Paris, Dunod.

Darnhoefer, I., Bellon, S., Dedieu, B., Milestad, R., 2010. Adaptiveness to enhance the sustainability of farming systems, A review, Journal of agronomy for sustainable development, 30, 545-555.

Dedieu, B., Chia, E., Leclerc, B., Moulin, C.-H., Tichit, M. (Eds.), 2008. L'élevage en mouvement-Flexibilité et adaptation des exploitations d'herbivores, Paris, Quae.

Desriers, M., 2007. L'agriculture française depuis cinquante ans : des petites exploitations familiales aux droits à paiement unique, Agreste Cahiers, 2, 3-14.

Desriers, M., 2011. Les productions se concentrent dans les exploitations spécialisées, Agreste Primeur, 272, 1-3.

Drouet, D., Boullet, P., Seronie-Vivien, J.-M., 2005. Le Concept d'exploitation agricole a-t-il un avenir ? Les Cahiers CER France, $32 \mathrm{p}$.

Fayol, H., 1918. Administration industrielle et générale, Paris, Dunod.

Gafsi, M., 1998. Les relations d'interdépendance entre l'exploitation agricole et son contexte local : une interprétation en termes de source de performance, Études et Recherches sur les Systèmes Agraires et le Développement, 31, 239-257.

Gafsi, M., 2003. Multifonctionnalité de l'agriculture et redéfinition du rapport de l'exploitation agricole au territoire, in Barthélemy, D., Delorme, H., Losch, B., Moreddiu, C., Nieddu, M., (Eds), La multifonctionnalité de l'activité agricole et sa reconnaissance par les politiques publiques, Dijon, SFER-EducagriCirad, 745-763. 
Gafsi, M., 2006. Exploitation agricole et agriculture durable, Cahiers Agricultures, $15,6,491-497$.

Gafsi, M., Legagneux, B., Nguyen, G., 2002. Agriculture territorialisée et développement territorial : une analyse exploratoire des transformations du rapport de l'agriculture au territoire en Midi-Pyrénées, Communication au Colloque DADP, Montpellier 17-18/12/2002.

Gambino, M., Vert, J. (Cord.), 2012. Le monde agricole en tendances. Un portrait social prospectif des agriculteurs, Paris, ministère de l'Agriculture et de la Pêche.

Gervais, M., Servolin, C., Weil, J.,1965. Une France sans paysans, Paris, Seuil.

Guibert, M., Sili, M., Arbeletche, P., Pineiro, D., Grosso, S., 2011. Les nouvelles formes d'agriculture entrepreneuriale en Argentine et en Uruguay, Économies et Sociétés, $A G, 33,1813-1831$.

Guichard, M., Michaud, R., 1994. La stratégie à pas contés : Piloter l'entreprise agricole dans l'incertitude et dans la complexité, Dijon, Cnerta-Sed.

Hatchuel, A., 2000. Quel horizon pour les sciences de gestion ? Vers une théorie de l'action collective, in David, A., Hatchuel, A., Laufer, R., (Eds), Les nouvelles fondations des sciences de gestion, Paris, Vuibert, 7-43.

Hémidy, L., Mawime, F., Soler, L.G., 1993. Instrumentation et pilotage stratégique dans l'entreprise agricole, Cahiers d'Économie et Sociologies Rurales, 28, 91-118.

Lamarche, H., 1991. L'agriculture familiale : une réalité polymorphe, Paris, L'Harmattan.

Landais, E., 1998. Agriculture durable : les fondements d'un nouveau contrat social ? Courrier de l'environnement de l'INRA, 33, 5-22.

Laurent, C., Maxime, F., Mazé, A., Tichit, M, 2003. Multifonctionnalité de l'agriculture et modèles de l'exploitation agricole, Économie Rurale, 273-274, 134152.

Laurent, C., Rémy, J., 2000. L'exploitation agricole en perspective, Courrier de l'environnement de l'INRA, 41, 5-22.

March, J.-G., Simon, H.-A., 1964. Organisation, problèmes psycho-sociologiques, Paris, Dunod.

Marshall, E., 1984, Gestion et enseignement de la gestion des exploitations agricoles, Éducation permanente, 77, 53-75.

Mignon, S., 2009. La pérennité organisationnelle : un cadre d'analyse, Revue Française de Gestion, 192, 73-89.

Mondy, B., Vincq, J.-L., Gafsi, M., 2012. Coopératives de diversification : création d'emplois, maintien d'exploitations et re-territorialisation de l'agriculture, Communication au Colloque SFER "Diversité et durabilité des modèles agricoles coopératifs dans un contexte de crises de la mondialisation" 6-7 novembre 2012, Paris.

Morgan, G., 1989. Images de l'organisation, Laval, Presses Universitaires de Laval.

Muller, P., Gerbaux, F., Faure, A., 1989. Les entrepreneurs ruraux : agriculteurs, artisans, commerçants, élus locaux, Paris, L'Harmattan.

Muller, P., Pernet, F., Le Monnier, J., 1984. Les agricultures différentes, Grenoble, La pensée sauvage. 
Nguyen, G., Gafsi, M., Legagneux, B., 2004. Exploitation agricole et développement territorial : quelles relations les exploitations entretiennent-elles avec leurs territoires ? Communication au colloque SFER, Paris 18-19 novembre 2004.

Osty, P.-L., 1978. L'exploitation agricole vue comme un système, Bulletin technique d'information, 326, 43-49.

Pernet, F., 1990. Exploitation agricole ou exploitation rurale ? in Coulomb, P., Delorme, H., Hervieu, B., Jollivet, M., Lacombe, P. (dir), Les agriculteurs et la politique, Paris, Presse de la Fondation nationale des sciences politiques, 301-309.

Petit, M., 1975. Évolution de l'agriculture et caractère familial des exploitations agricoles, Économie Rurale, 106, 45-55.

Petit, M., 1981. Théorie de la décision et comportement adaptatif des agriculteurs, Actes du Séminaire "Formation des agriculteurs et apprentissage de la décision », Dijon, 21 janvier 1981.

Petit, M., 2006. L'exploitation agricole familiale : leçons actuelles et débats anciens, Cahiers Agricultures, 15, 6, 486-490.

Plane, J.-M., 2000. Théorie des organisations, Paris, Dunod.

Rémy, J., 2013. L'exploitation agricole : une institution en mouvement, Déméter, 357-384.

Reynaud, E. (Ed.), 2006. Le développement durable au cour de l'entreprise, Paris, Dunod.

Rojot, J, Bergmann, A., 1989. Comportement et organisation, Paris, Vuibert,

Sebillotte, M., 1996. Les mondes de l'agriculture : une recherche pour demain, Paris, INRA Éditions.

Séronie, J.-M., 2007. L'exploitation agricole flexible, Les Cahiers CER France.

Sorge, A., 1997. "Organization behaviour " in Sorge A., Warner M. (Eds), The handbook of organizational behaviour, New York, Thomson Business Press.

Taylor, F.W., 1911, The principles of scientific management, New York, Harper and Brothers.

Tchayanov A., 1925. L'organisation de l'économie paysanne, Paris, Librairie du Regard.

Tonneau, J.-P., De Aquino, J.-R., Teixeira, O.-A., 2005. Modernisation de l'agriculture familiale et exclusion : le dilemme des politiques agricoles, Cahiers Agricultures, 14, 1, 30-34.

Vandenbroucke, P., 2013. Transformation de l'unité de production agricole : d'une exploitation sectorielle à une exploitation agricole territoriale, Thèse de Doctorat en Géographie, Aménagement et Urbanisme, Université de Lyon 2.

Wernerfelt, B., 1995. The resources-based view on the firm : ten years after, Strategic Management Journal, 16, 3, 171-174. 